\title{
EMR Spectra Thin Films Doped with High Concentration of Co and Cr on Quartz and Sapphire Substrates
}

\author{
B. CienieK*, I. Stefaniuk And I. VirT
}

Faculty of Mathematics and Natural Sciences, University of Rzeszów, S. Pigonia 1, 35-959 Rzeszów, Poland

\begin{abstract}
We have studied magnetic properties of zinc-oxide composite doped with high concentration (up to 20\%) of Co and $\mathrm{Cr}$ ions. The pulsed laser deposition method was used to obtain samples on quartz glass and sapphire substrates. Samples were annealed at $100-250{ }^{\circ} \mathrm{C}$ for $\mathrm{ZnO}$ on quartz substrate, and $300-700{ }^{\circ} \mathrm{C}$ on sapphire substrate. EMR measurements were carried out and temperature dependence of the EMR spectra was obtained. The angular dependence in two samples orientation, vertical and horizontal, were also obtained. Analysis of the temperature dependences of the integral intensity of EMR spectra was carried out using the Curie-Weiss law.
\end{abstract}

DOI: 10.12693/APhysPolA.132.30

PACS/topics: 68.35.bg, 75.30.Gw, 76.30.-v

\section{Introduction}

Diluted magnetic semiconductors (DMS) are alloys where a stoichiometric fraction of the constituent atoms has been replaced by magnetic transition metal atoms. Based on mean-field models it was shown that wide band gap diluted semiconductors could lead to room temperature ferromagnetism [1]. Due to its rare physical properties, such as energy gap of about $3.4 \mathrm{eV}$ at room temperature and the exciton binding energy of about $60 \mathrm{meV}$, zinc oxide (chemical formula $\mathrm{ZnO}$ ) is used for devices based on semiconductor structures [2,3], such as varistors [4] or sensors [5]. In additional, relatively simple production technology of $\mathrm{ZnO}$ crystals gives us hope to lower cost of production equipment based on $\mathrm{ZnO}$ in comparison to the popular GaN. This, together with the properties such as transparency in visual region and piezoelectricity have generated huge interest on $\mathrm{ZnO}$ based DMS $[6,7]$. DMS are of interest for study for their unique spintronics properties with possible technological applications [810], such as spin-spin exchange interaction between the localized magnetic moments and the band electrons [11]. Some subsequent theoretical using density functional theory (DFT) $[12,13]$ and experimental $[14,15]$ works show that $n$-type Co-doped $\mathrm{ZnO}$ also possesses room temperature ferromagnetism.

Using a modified Zener model, Dietl et al. predicted that $\mathrm{ZnO}$ based DMS could lead the transition temperature to much higher than the room temperature [16]. According to this theory, $p-d$ interactions are the reason for long-range magnetic coupling. However, the investigated magnetic $\mathrm{ZnO}$ samples are either $n$-type conducting or insulating. The observed weak ferromagnetism in implanted $\mathrm{ZnO}$ containing ferromagnetic nanocrystals and in PLD grown $\mathrm{ZnO}$ with diluted magnetic ions is due to ferromagnetic nanocrystals and the acceptor-like defects [17], respectively. For the diluted $\mathrm{ZnO}: \mathrm{Co}$ and

*corresponding author; e-mail: cieniek@if.univ.rzeszow.pl
$\mathrm{ZnO}: \mathrm{Cr}$, it would be interesting to check if there is interaction between free electrons and $d$-electrons in Co and $\mathrm{Cr}$ ions, and if this $s-d$ interaction results in ferromagnetic coupling.

Some groups have reported ferromagnetism in $\mathrm{ZnO}$ doped with transition metal systems Curie temperatures $T_{C}$ ranging from 30 to $550 \mathrm{~K}[15,18-23]$, while others have found antiferromagnetic, spin glass, or paramagnetic behaviour [7, 24, 25].

The existence of ferromagnetic ordering in $(\mathrm{Zn}, \mathrm{Co}) \mathrm{O}$, has been theoretically proposed to be attributed to the double exchange interaction [6] or the RudermanKittel-Kasuya-Yosida (RKKY) interaction between Co ions [26]. Later calculation showed that the ground state of Co-doped $\mathrm{ZnO}$ is spin glass without doping due to the short range interactions between transition metal atoms [27].

We report here the magnetic properties of zinc-oxide composite $(\mathrm{ZnO})$ doped with $\mathrm{Co}$ and $\mathrm{Cr}$ ions. Electron magnetic resonance (EMR) spectra have been measured and analyzed to extract information on the characteristics of the incorporation of the ions in the lattice.

\section{Experiment}

Hard solutions of $\mathrm{Zn}_{1-x} \mathrm{Co}_{x} \mathrm{O}$ and $\mathrm{Zn}_{1-x} \mathrm{Cr}_{x} \mathrm{O}$ were obtained by the method of solid state reactions, which is widely applied in ceramic technology. Materials of special cleanness were used as initial components for preparation of charge. The powders of connections $\mathrm{CoCO}_{3}$ and $\mathrm{CrCO}_{3}$ obtained by growing shallow to the size of particles 50-100 nm, and mixed up with powder $\mathrm{ZnO}$ and small amount of water in the jasper drums of planetary mill SAND-1-1. Time of mixing and grating was determined by the degree of homogenization and was set to $16 \mathrm{~h}$.

The mixture was drained in temperature $120 \pm 5^{\circ} \mathrm{C}$. After that the mixture was annealing in air at $700 \pm 5^{\circ} \mathrm{C}$ for $4 \mathrm{~h}$. Press-purveyances by a diameter $11.5-15 \mathrm{~mm}$ thick formed 1-2.5 $\mathrm{mm}$ by applying pressure of 40$60 \mathrm{MPa}$ on a hydraulic press PG-10 without the use 
of plastificators. Mixtures were annealed at temperatures near to $1000^{\circ} \mathrm{C}$. Hard solutions of $\mathrm{Zn}_{1-x} \mathrm{Co}_{x} \mathrm{O}$ and $\mathrm{Zn}_{1-x} \mathrm{Cr}_{x} \mathrm{O}$ with $x=0.2$ and $x=0.04$ were obtained in this way. Standards were annealed in the chamber stove of periodic action VTP-06M1 in air (the accuracy of temperature control was $\pm 5^{\circ} \mathrm{C}$ ) during $3 \mathrm{~h}$. Maximal temperature of annealing, which replies the isothermal area of curve of heating-cooling, was $1110^{\circ} \mathrm{C}$.

Technology of preparation of ceramic materials on the basis of $\mathrm{ZnO}$, as remarked earlier, utilizes solid states reactions. The reactions $\mathrm{CoCO}_{3}+\mathrm{ZnO}>\mathrm{Zn}_{1-x} \mathrm{Co}_{x} \mathrm{O}$ and $\mathrm{CrCO}_{3}+\mathrm{ZnO}>\mathrm{Zn}_{1-x} \mathrm{Cr}_{x} \mathrm{O}$ yield powders of $\mathrm{Zn}_{1-x} \mathrm{Co}_{x} \mathrm{O}$ and $\mathrm{Zn}_{1-x} \mathrm{Cr}_{x} \mathrm{O}$.

Samples of zinc-oxide doped with cobalt and chromium from solutions above, were obtained by Pulsed Laser Deposition (PLD) method. The substrate temperature was $25^{\circ} \mathrm{C}$ for quartz, and $200{ }^{\circ} \mathrm{C}$ for sapphire. Beam parameters: $0.2 \mathrm{~mm}, 0.5 \mathrm{~J}$ in $10 \mathrm{~ns}$ pulse, Gaussian distribution, repetition frequency $0.3 \mathrm{~Hz}$. Time of layer growth was about 30 minutes. The layer thickness is from 300 to $500 \mathrm{~nm}$. Samples were annealed in air. Compared to our previous work [28] with $\mathrm{ZnO}$, samples with cobalt were received in different substrate temperature and with different thickness. EMR measurements were performed in the X-band on Bruker multifrequency and multiresonance FT-EPR ELEXSYS E580 spectrometer. The temperature of the samples was controlled in the range of 95-300 K using the Bruker liquid N gas flow cryostat with 41131 VT digital controller, and in the range of 4$300 \mathrm{~K}$ using the Oxford Instruments ESR 900 liquid He gas flow cryostat with MercuryITC digital controller.

\section{Results and discussion}

EMR spectra comparison for the annealed and non annealed sample of $\mathrm{ZnO}: \mathrm{Co}$ and $\mathrm{ZnO}: \mathrm{Cr}$ are similar and were analyzed. Samples on sapphire substrate in contrast to quartz have additional narrow line shown in Fig. 1. This line is coming from chrome inside substrate. We are interested more in magnetic properties of $\mathrm{ZnO}$ layer instead of substrate, that is why further in the work we focused on broad line around $350 \mathrm{mT}$. In the spectrum low-field strongly asymmetric line can be observed whose analysis will be made in a separated work. Anisotropy of EMR spectra of $\mathrm{ZnO}$ : $\mathrm{Cr}$ layer on sapphire substrate is shown in Figs. 2 and 3.

Effective spectroscopic $g$-factor and the peak-to-peak line width $\left(H_{p p}\right)$ of the wide resonance line near $350 \mathrm{mT}$ were determined. Based on those parameters the CurieWeiss temperature was determinate. In addition, temperature dependence of intensity and peak-to-peak line width are shown in Fig. 4. Since the broad EMR line is asymmetric, the accuracy of parameters measured directly from the experimental spectrum is rather limited. Therefore, additionally the experimental line was fitted using the Lorentzian type curve, since such curves describe satisfactorily experimental EMR lines of DMS with manganese in high temperature range (see [6] and references therein). In this way we determined parameters for

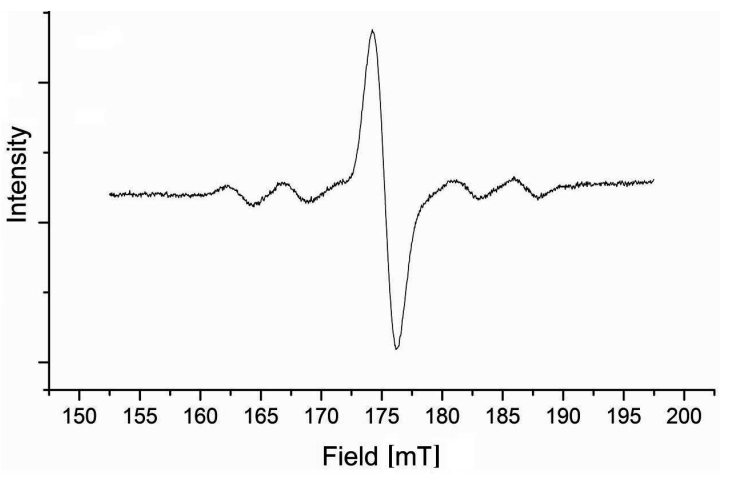

Fig. 1. EMR spectra of narrow Cr line from sapphire, $\mathrm{ZnO}: \mathrm{Cr}$ annealed at $300^{\circ} \mathrm{C}$ measured at temperature of $93 \mathrm{~K}$.

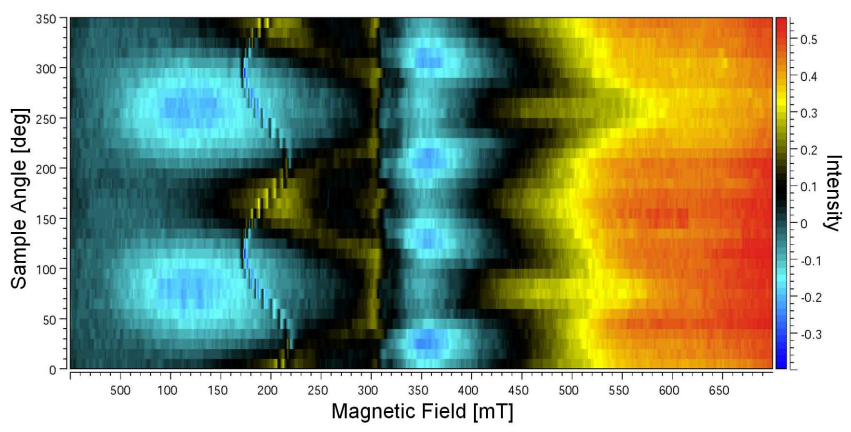

Fig. 2. Angular dependence of vertical orientation for $\mathrm{ZnO}: \mathrm{Cr}$ sapphire sample annealed at $700 \mathrm{~K}$.

EMR lines such as the peak-to-peak line width $\left(H_{p p}\right)$, the intensity $(I)$ as well as the resonance field $\left(H_{r}\right)$. Based on these data we obtained the temperature dependences of gyromagnetic factor $g(T)$ and integral intensity. At fitting the Lorentz type curve the lower field part of EMR line was included because of its regular nature.

We used the Curie-Weiss law to analyze the temperature dependences of the integral intensity, which is directly proportional to the magnetic susceptibility $\chi$. A linear increase of $\chi^{-1}(T)$ at higher temperatures can be fitted to the Curie-Weiss law [29, 30]:

$$
\left(\chi(T)-\chi_{0}\right)^{-1}=\left(T-\theta_{\mathrm{CW}}\right) / C,
$$

where $C$ is the Curie constant, $\theta_{\mathrm{CW}}$ is the paramagnetic

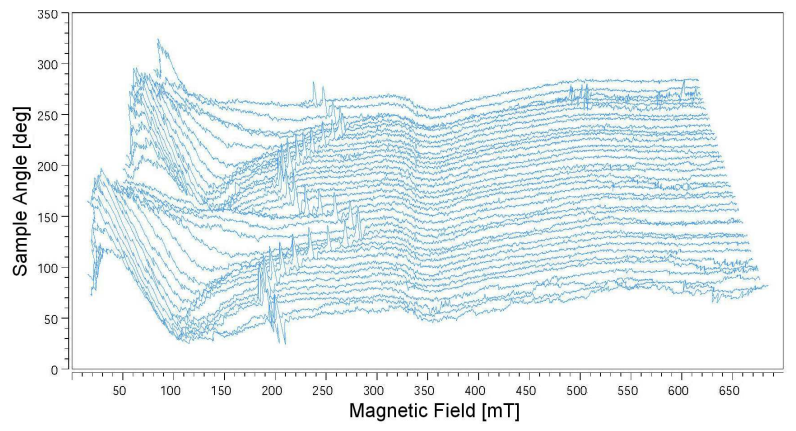

Fig. 3. Angular dependence of horizontal orientation for $\mathrm{ZnO}$ :Cr sapphire sample annealed at $500 \mathrm{~K}$. 


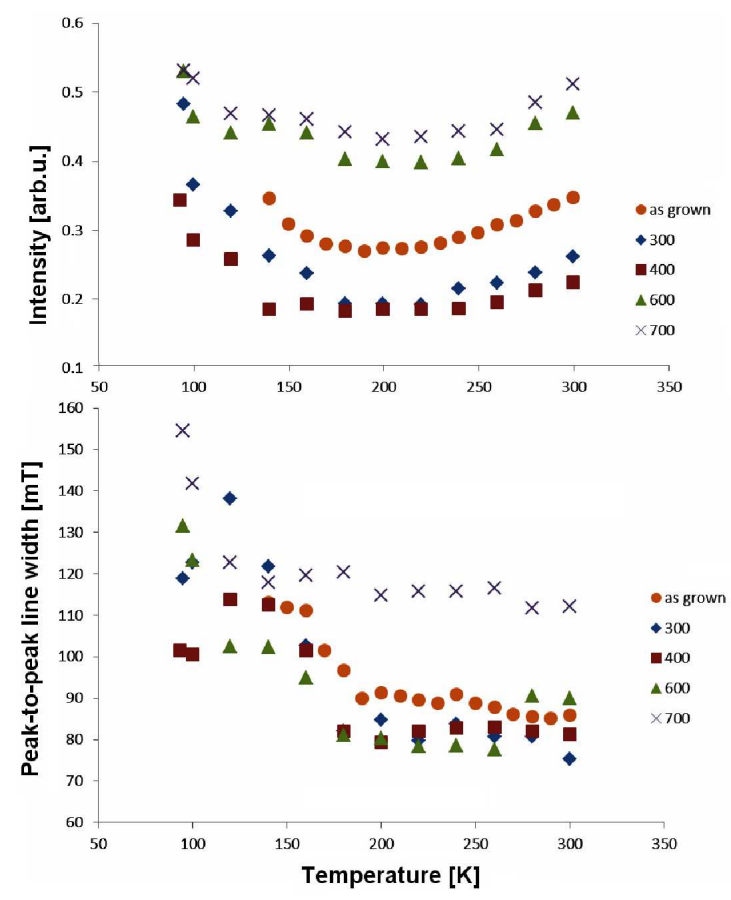

Fig. 4. Temperature dependence of intensity (top) and peak-to-peak line width (bottom) of $\mathrm{ZnO}$ :Co samples on quartz substrate.

Curie-Weiss temperature of all samples.

TABLE I

\begin{tabular}{c|c|c}
\hline \hline & Temperature $[\mathrm{K}]$ & $\theta_{\mathrm{CW}}$ \\
\hline \multicolumn{3}{c}{ Samples on quartz substrate } \\
\hline ZnO:Cr annealed & 136.2 & $3.33 \times 10^{5}$ \\
ZnO:Cr & 138.3 & $2.00 \times 10^{5}$ \\
ZnO:Co & 165.5 & $1.00 \times 10^{5}$ \\
ZnO:Co annealed & 150.8 & $1.43 \times 10^{5}$ \\
\hline \multicolumn{3}{c}{ Samples on sapphire substrate } \\
\hline ZnO:Cr as grown & 94.5 & $2.16 \times 10^{5}$ \\
ZnO:Cr 300 K annealed & 109.6 & $1.02 \times 10^{5}$ \\
ZnO:Cr 400 K annealed & 86.9 & $1.24 \times 10^{5}$ \\
ZnO:Cr 600 K annealed & 50.3 & $3.78 \times 10^{5}$ \\
ZnO:Cr 700 K annealed & 44.0 & $5.93 \times 10^{5}$
\end{tabular}

Curie temperature, and $\chi_{0}$ is a temperature independent term to account for the diamagnetic host and any Pauli paramagnetism contribution. Fitting yields the following values shown in Table I.

\section{Conclusions}

For annealed and non annealed layers on quartz substrate observed characteristic Curie-Weiss temperature around $200 \mathrm{~K}$ in which all EMR line parameters such as: peak-to-peak line width $\left(H_{p p}\right)$, the intensity $I$ as well as the gyromagnetic factor $g(T)$ are changed. For samples on sapphire substrate the Curie-Weiss temperature decreases with increase of the annealing temperature, which is shown in Fig. 5.

In summary, we have reported the X-band EMR studies of $\mathrm{ZnO}$ : $\mathrm{Co}$ and $\mathrm{ZnO}$ :Cr. We have determined from the

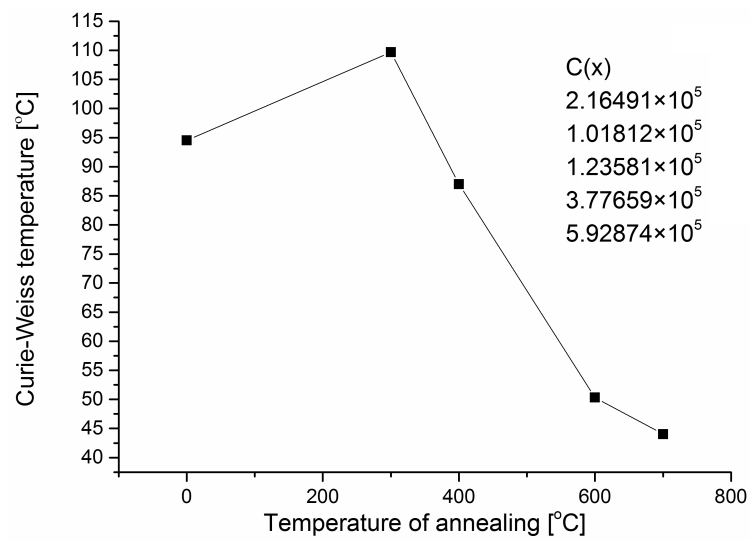

Fig. 5. Curie-Weiss temperature dependence of the temperature of annealing for $\mathrm{ZnO}: \mathrm{Cr}$ samples on sapphire substrate.

EMR lines the parameters: the peak-to-peak line width $H_{p p}$, the intensity $I$ as well as the resonance field $H_{r}$. The results of temperature dependence of EMR spectra for the samples and linear extrapolations to the CurieWeiss law indicate the ferromagnetic interaction between $\mathrm{Co}$ and $\mathrm{Cr}$ ions characterized by the Curie temperatures from $44.0 \mathrm{~K}$ to $109.6 \mathrm{~K}$ and from $136.2 \mathrm{~K}$ to $165.5 \mathrm{~K}$, respectively.

In this study the magnetic properties of $\mathrm{Cr}$ and $\mathrm{Co}$ with a high concentration up to $20 \%$ were analyzed. The line in a broad asymmetric Dyson shape was observed, consisting of two different lines staggered. We have determined from the EMR lines the parameters: the peakto-peak line width $H_{p p}$, the intensity $I$ as well as the resonance field $H_{r}$. The results of temperature dependence of EMR spectra for the samples and linear extrapolations to the Curie-Weiss law indicate the ferromagnetic interaction between $\mathrm{Co}$ and $\mathrm{Cr}$ ions.

With increase of the annealing temperature we observe stronger paramagnetic properties as a result from weakening the exchange coupling between $\mathrm{Cr}$ and $\mathrm{Co}$ ions. A similar effect was observed at work [24] for ZnO:Co layers, with $\mathrm{Co}=25 \%$. The mechanism responsible for the observed ferromagnetism is still not clear, and the source of the ferromagnetism remains controversial.

By analyzing the EMR linewidth behavior, similar as in previous paper [30] for low concentration of ions, we claim that a combined effect of the exchange and dipolar broadening plays an important role in the mechanism of linewidth formation in those materials.

\section{References}

[1] T. Dietl, J. Phys. Condens. Matter 19, 165204 (2007).

[2] J. Weng, Y. Zhang, G. Han, Y. Zhang, L. Xu, J. Xu, X. Huang, K. Chen, Thin Solid Films 478, 25 (2005).

[3] J. Wilkinson, K.B. Ucer, R.T. Wiliams, Radiat. Meas. 38, 501 (2004).

[4] K.X. Ya, H. Yin, T.M. De, T.M. Jing, Mater. Res. Bull. 33, 1703 (1998). 
[5] J. Xu, Q. Pan, Y. Shun, Z. Tian, Sensor. Actuat. B Chem. 66, 277 (2000).

[6] N. Samarth, J.K. Furdyna, Phys. Rev. B 37, 9227 (1988).

[7] N. Volbers, H. Zhou, C. Knies, D. Pfisterer, J. Sann, D.M. Hofmann, B.K. Meyer, Appl. Phys. A Mater. 88, 153 (2007).

[8] Semiconductors Spintronics and Quantum Computation, Eds. D.D. Awschalom, D. Loss, N. Samarth, Springer, New York 2002.

[9] J.K. Furdyna, J. Appl. Phys. 64, R29 (1988).

[10] H. Ohno, Science 281, 951 (1998).

[11] W. Dobrowolski, J. Kossut, T. Story, in: Handbook of Magnetic Materials Vol. 15, Ed. K.H.J. Bushow, Elsevier, Netherlands 2003, p. 289.

[12] K. Sato, H. Katayama-Yoshida, Jpn. J. Appl. Phys. 39, L555 (2000).

[13] K. Sato, H. Katayama-Yoshida, Jpn. J. Appl. Phys. 40, L334 (2001).

[14] W. Prellier, A. Fouchet, B. Mercey, Ch. Simon, B. Raveau, Appl. Phys. Lett. 82, 3490 (2003).

[15] K. Ueda, H. Tabata, T. Kawai, Appl. Phys. Lett. 79 , 988 (2001).

[16] T. Dietl, H. Ohno, F. Matsukura, J. Cibert, D. Ferrand, Science 287, 1019 (2000).

[17] Q. Xu, H. Schmidt, S. Zhou, K. Potzger, M. Helm, H. Hochmuth, M. Lorenz, A. Setzer, P. Esquinazi, C. Meinecke, M. Grundmann, Appl. Phys. Lett. 92, 082508 (2008).
[18] S.W. Jung, S.-J. An, G.-C. Yi, C.U. Jung, S.-I. Lee, S. Cho, Appl. Phys. Lett. 80, 4561 (2002).

[19] D.A. Schwartz, N.S. Norberg, Q.P. Nguyen, J.M. Parker, D.R. Gamelin, J. Am. Chem. Soc. 125, 13205 (2003)

[20] H.-J. Lee, S.-Y. Jeong, C.R. Cho, C.H. Park, Appl. Phys. Lett. 81, 4020 (2002).

[21] Y.M. Cho, W.K Choo, H. Kim, D. Kim, Y.E. Ihm, Appl. Phys. Lett. 80, 3358 (2002).

[22] P. Sharma, A. Gupta, K.V. Rao, F.J. Owens, R. Sharma, R. Ahuja, J.M. Osorio Guillen, B. Johansson, G.A. Gehring, Nat. Mater. 2, 673 (2003).

[23] T. Wakano, N. Fujimura, Y. Morinaga, N. Abe, A. Ashida, T. Ito, Physica E 10, 260 (2001).

[24] J.H. Kim, H. Kim, D. Kim, Y.E. Ihm, W.K. Choo, J. Appl. Phys. 92, 6066 (2002).

[25] A.O. Ankiewicz, M.C. Carmo, N.A. Sobolev, W. Gehlhoff, E.M. Kaidashev, A. Rahm, M. Lorenz, M. Grundmann, J. Appl. Phys. 101, 024324 (2007).

[26] A.F. Jalbout, H. Chen, S.L. Whittenburg, Appl. Phys. Lett. 81, 2217 (2002).

[27] E.-C. Lee, K.J. Chang, Phys. Rev. B 69, 085205 (2004).

[28] B. Cieniek, I. Stefaniuk, I. Virt, Nukleonika 58, 359 (2013).

[29] J.S. Dyck, Č. Drašar, P. Lošt'ák, C. Uher, Phys. Rev. B 71, 115214 (2005).

[30] D.L. Huber, G. Alejandro, A. Caneiro, M.T. Causa, F. Prado, M. Tovar, S.B. Oseroff, Phys. Rev. B 60, 12155 (1999). 